\title{
Applicability of MIMO to satellite communications
}

\author{
Jukka Kyröläinen $^{1, *, * *, \dagger}$, Ari Hulkkonen ${ }^{1}$, Juha Ylitalo ${ }^{1}$, Aaron Byman ${ }^{1}$, Bhavani Shankar ${ }^{2}$, \\ Pantelis-Daniel Arapoglou ${ }^{3}$ and Joel Grotz ${ }^{4, * *}$ \\ ${ }^{1}$ Elektrobit Wireless Communications Ltd, Tutkijantie 8, 90570 Oulu, Finland \\ ${ }^{2}$ University of Luxembourg, Kirchberg Campus, L-1359 Luxembourg, Luxembourg \\ ${ }^{3}$ ESA/ESTEC, Noordwijk, 2200 AG, the Netherlands \\ ${ }^{4}$ Chateau de Betzdorf, SES ASTRA TechCom, Betzdorf, 6815, Luxembourg, Luxembourg
}

\begin{abstract}
SUMMARY
This paper presents achievements of an on-going activity where the applicability of MIMO to satellite communications with Digital Video Broadcasting - Satellite services to Handhelds as the key application is studied. The potential satellite and hybrid satellite-terrestrial MIMO scenarios are described, and the applicable MIMO schemes for each scenario are selected. The performance of the MIMO schemes was studied by performing comprehensive computer simulations, and the main results are presented in this paper. Copyright (C) 2013 John Wiley \& Sons, Ltd.
\end{abstract}

Received 31 October 2012; Revised 14 June 2013; Accepted 24 June 2013

KEY WORDS: $\quad$ satellite communications; MIMO; multi-antenna systems; hybrid terrestrial-satellite communications; DVB-SH; broadband wireless radio access

\section{INTRODUCTION}

Since the early 1990s multi-antenna systems have been a hot research topic in wireless communications. In addition to adaptive antenna arrays, Multiple-Input and Multiple-Output (MIMO), which theoretically provided drastic improvement in the spectral efficiency and data rates of radio access networks, became one of the most important technologies supporting future's broadband wireless access. Due to the various optional techniques that different MIMO schemes offer (diversity, beam forming, pre-coding, and spatial multiplexing), MIMO techniques were widely adopted in evolving terrestrial wireless communication standards, from cellular mobile systems such as ETSI 3GPP Universal Mobile Telecommunications System and Long Term Evolution to broadband access such as WiFi (IEEE 802.11n) and WiMAX (IEEE 802.16e).

In addition to two-way communications, multiple transmit antennas have been considered in broadcasting systems such as Digital Video Broadcasting - Terrestrial Second Generation. Higher spectral efficiency and higher data rates allow more services to be provided in the same transport stream. In addition, increased reliability translates into higher robustness against the menacing channel fading.

Compared with the terrestrial domain, adoption of MIMO technology in the satellite community has been slow. Fixed satellite system links are based on highly directive high-gain dish antennas, and the gain obtained with multi-antenna techniques is marginal compared with system complexity and practical implementation issues. Spot beam technique deployed by mobile satellite system Geosynchronous Earth Orbit satellites employing large antennas is a practical option. A comprehensive review on the progress of MIMO technology in satellite communications can be found in [1]. As

*Correspondence to: Jukka Kyröläinen, Anite Telecoms, Tutkijantie 6, 90570 Oulu, Finland.

${ }^{\dagger}$ E-mail: jukka.kyrolainen@ anite.com

***Affiliation at the time of paper submission. 
discussed in [1], satellite communications are characterized by a specific operation environment in which radio channel properties differ significantly from those in terrestrial wireless radio access systems. For example, line-of-sight connection is usually needed to guarantee adequate link budget for reliable data transmission. Moreover, the satellite radio channel does not have rich multi-path propagation characteristics due to the absence of reflectors near the satellite. This is a specific challenge from the viewpoint of MIMO transmission in the spatial multiplexing (SM) mode.

Whereas the terrestrial systems are suitable for utilizing mainly the spatial domain to induce multiple streams, utilization of polarization dimension is an attractive alternative for satellite systems due to the abovementioned Line-of-sight (LOS) connections. The feasibility of deploying dual-polarized antennas for communicating with a mobile terminal has been shown to be amenable for MIMO [1]. Extending the use of MIMO techniques for hybrid terrestrial/ satellite systems have been also considered $[2,3,5]$.

\section{DESCRIPTION OF DVB-SH SYSTEM}

Digital Video Broadcasting - Satellite services to Handhelds (DVB-SH) standard [10, 11] is one of the first comprehensive standardization efforts in the area of hybrid satellite-terrestrial communications. The DVB-SH is aiming at providing ubiquitous Internet Protocol based multimedia services to a variety of mobile and fixed terminals having compact antennas with very limited directivity at frequencies below $3 \mathrm{GHz}$. The DVB-SH system is based on a hybrid architecture combining the satellite component (SC) and the complementary ground component (CGC). The terrestrial repeaters provide local retransmission, on the overlapping frequency in case of single-frequency network configuration (SFN) or on a non-overlapping frequency in case of multi-frequency network configuration (MFN). The waveform of the SC transmission can be either orthogonal frequency division multiplexing (OFDM) in the SH-A mode or time division multiplexing (TDM) in the SH-B mode, whereas the CGC uses always the OFDM waveform. The SFN configuration is possible only in the SH-A mode, whereas the MFN configuration is possible for both the SH-A and SH-B modes. The physical layer provides protection against long fading states of satellite channel by employing a time interleaver, which has influence length that can expand up to $10 \mathrm{~s}$. The physical layer forward error correcting (FEC) channel coding scheme is the 3GPP2 Turbo coding with different puncturing patterns for code rate adjustment. The supported FEC code rates range from $1 / 5$ to $2 / 3$. The FEC and time interleaving blocks are similar for both transmission modes. Other common processing blocks are mode adaptation and stream adaptation. The mode adaptation consists of cyclic redundancy check encoding, to provide error detection on every MPEG packet, and of inserting an Encapsulation Signaling. While the current version of the air interface fully supports only MPEG-TS input stream only, mode adaptation is able to provide support for flexible stream format. Stream adaptation provides padding to complete a constant length Encapsulation Frame EFRAME and performs scrambling. EFRAME length is designed to match the turbo code block size. A block diagram of DVB-SH transmitter architecture is shown in Figure 1.

In addition to the physical layer channel coding and time interleaving, the DVB-SH standard employs a link layer SH-specific FEC, named iFEC, which can span over tens of seconds. After the common processing blocks, the transmitter chains of the OFDM and TDM modes are differentiated. The OFDM blocks are briefly described in the following. The bit demultiplexing combines the hierarchical transmission streams into one stream if hierarchical modulation is applied. The symbol interleaving is performed symbol wise in frequency domain before mapping the bits into modulation symbols. After modulation, the data stream is organized into time-frequency grid (framing) to form frequency domain data for each OFDM symbol. The pilots and transmission parameter signaling (TPS) symbols are added into the time-frequency slots reserved for that use. Finally, the frames are passed to inverse fast Fourier transform (IFFT) block, which converts the frequency domain data into time domain signal and adds cyclic prefix. Supported modulation schemes for OFDM are Quadrature Phase Shift Keying (QPSK) and 16 quadrature amplitude modulation (16QAM). The transmit processing of TDM is simpler with the interleaved bits along with header information (frame size and interleaver parameters) 


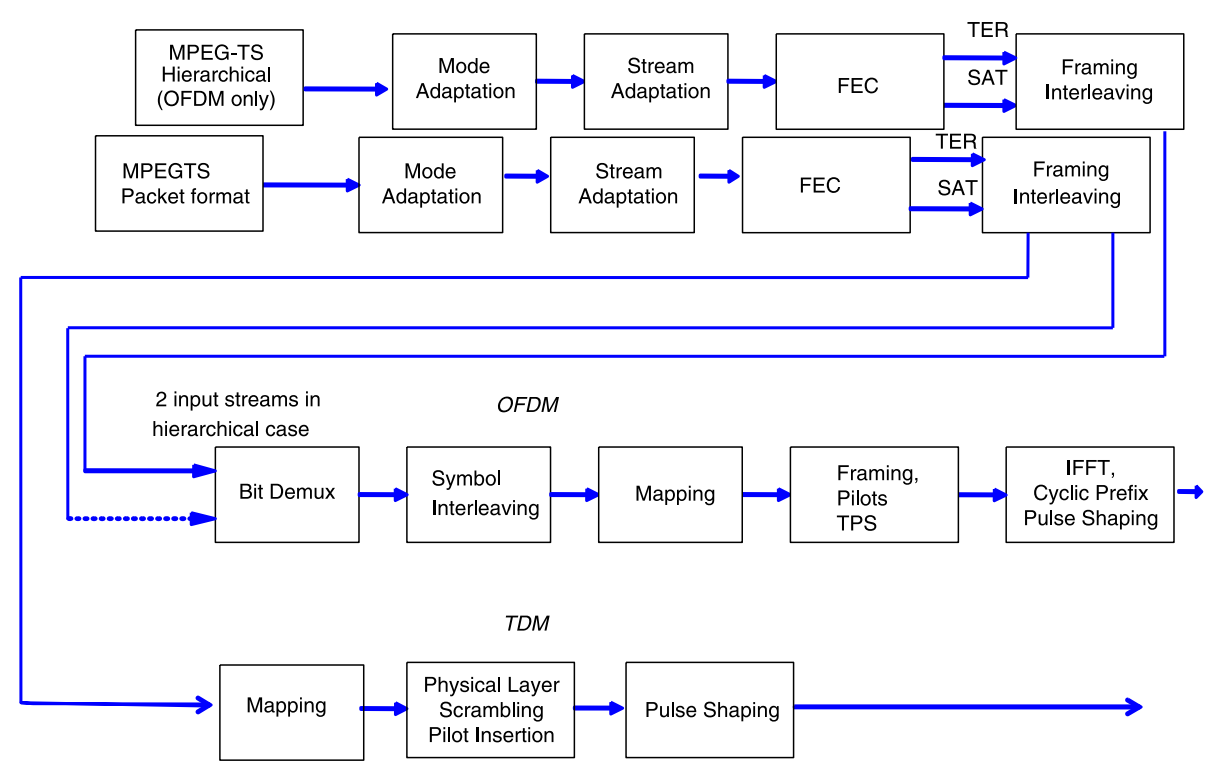

Figure 1. Digital Video Broadcasting - Satellite services to Handhelds transmitter block diagram. OFDM, orthogonal frequency division multiplexing; FEC, forward error correcting; IFFT, inverse fast Fourier transform; TPS, transmission parameter signaling; TDM, time division multiplexing.

and padding bits modulated using QPSK, 8PSK or 16APSK. The resulting symbols are packed to a frame whose data-carrying capacity should match that of the OFDM frame in hybrid mode operation. The resulting stream is scrambled using a complex pseudo-random sequence before being input to pulse shaping and radio frequency conversion. Two receiver classes are defined. Class 1 has short physical layer time interleaver $(200 \mathrm{~ms})$, whereas class 2 receiver supports full length physical layer interleaving. Class 1 transmission is often combined with link layer iFEC.

\section{MIMO EXTENSION TO DVB-SH}

In the first phase of this study, potential MIMO configurations and MIMO coding schemes were evaluated in order to select the feasible simulation scenarios and MIMO methods. The target was not only to find out the feasible methods for an elaborate simulation campaign but also to keep the complexity of user devices at a reasonable level. The fundamental criteria for the selection of MIMO methods for DVB-SH hybrid satellite system are the following: (1) The reception must be possible also in cases that only one of the links (satellite or terrestrial link) is available. Therefore, it is not possible to multiplex spatial streams over the distributed links. (2) The land mobile satellite (LMS) radio channel is usually not a rich multipath propagation environment. (3) The user terminal (UT) antenna separation cannot be large enough - particularly for handheld terminals - to obtain low spatial correlation between the antennas in LMS channel. (4) DVB-SH being a broadcast service, the transmitter cannot exploit channel state information for adapting the MIMO coding to channel conditions. Based on 2 and 3 , it is obvious that the MIMO-antenna system with co-located antennas on orthogonal polarizations is the most plausible antenna configuration for the DVB-SH MIMO extension. Four MIMO scenarios were identified and taken up for further evaluation.

\section{Scenario 1: satellite only $2 \times 2$ MIMO}

This scenario consists of a single GEO satellite with two orthogonally polarized (right-hand circular polarization (RHCP)/left-hand circular polarization (LHCP)) Tx-antennas set up as a dual polarization per beam payload and a UT with two co-located orthogonally polarized (RHCP/LHCP) receiver $(\mathrm{Rx})$-antennas. The selection of the MIMO schemes for this scenario was carried out on the basis 
of knowledge from various sources. A number of MIMO techniques have been developed for terrestrial $2 \times 2$ MIMO systems. This definitely provides for a first choice to look for albeit fundamental differences between spatial multipath MIMO channels and the dual polarization LMS channel exist. Due to the increased interest in a possible application of dual polarization MIMO to mobile satellite broadcasting systems, a number of publications have been recently produced concerning different aspects of a purely dual polarization MIMO LMS [1]. These are extremely valuable to the present activity and will serve as a guide for the final choice of MIMO techniques. Past MIMO related European Space Agency (ESA) activities provide also important information for the MIMO technique selection. Results on MIMO performance from applicable wireless standards, particularly Digital Video Broadcasting - Next Generation Handheld (DVB-NGH), were also taken into account. DVB-NGH is the extension of the DVB-H standard concerning the broadcasting of TV to highspeed terminals published in 2012 including a satellite and a hybrid profile [6]. Based on these sources and theoretical analysis, four coding schemes were assessed via simulations. These are 2xSISO, Alamouti transmit diversity, SM and the Golden code. 2xSISO provides multiplexing gain through a simple extension of current DVB-SH SISO standard. The transmission is multiplexed on two antennas, and the two streams are independently decoded at the receiver. This scheme relies on assumption that the cross-polarization ratio (XPR) of the LMS channel is very high, and therefore, the interference between the streams remains at an acceptable level. The Alamouti space-time block coding (STBC) provides full diversity gain but no multiplexing gain. This method was selected because of its low complexity through linear single symbol decodability and because of the possibly valuable diversity gain in the sensitive satellite channels. The SM was selected as a full rate spacetime code to exploit joint-decoding of two symbols within one symbol time in order to achieve multiplexing gain. The theoretical advantages of full-rate full-diversity (FR-FD) codes provide a strong suggestion to include FR-FD codes among the set of simulation codes. However, according to previous studies, it was expected that the performance of FR-FD codes may not be superior compared with other schemes in presence of FEC and long interleaving combined with LMS channel. However, despite of its high complexity, Golden code was selected as a reference to represent the optimal FR-FD code.

\section{Scenario 2: hybrid satellitelterrestrial $2 \times 1 / 2 \times 2$ MIMO}

This is a basic hybrid transmission scheme with one satellite (SC) and one terrestrial base station or repeater (CGC) jointly transmitting data to users. The SC and CGC are transmitted on orthogonal polarizations. One stream is transmitted per link and SFN configuration is considered. From an architectural point of view, the benchmark for Scenario 2 is the hybrid SISO equivalent to today's DVB-SH. The UT is equipped with either a single-polarization antenna or a dual-polarized antenna, which may be either circular or linear. A single-polarization antenna at UT leads to a $2 \times 1$ multiple-input singleoutput system. Use of dual-polarized antennas at UT leads to a $2 \times 2$ MIMO system. It must be noted that only for a small percentage of the coverage both the SC and the CGC will be available. The MIMO scheme selection for this scenario was performed based on literature study and insights from standardization perspective. A setting similar to Scenario 2 has been standardized in DVB-NGH forum [6] and a recent ITU-R report proposes certain techniques for use in hybrid/integrated systems operating in the $1-3 \mathrm{GHz}$ band [7]. Furthermore, by taking into account the requirement of the ability to decode the transmission in case of a failure of either of the links, it is not possible to increase the spatial rate by multiplexing streams over the links. Therefore, the Alamouti STBC over the links is the only practical choice for this scenario.

\section{Scenario 3: hybrid satellite/terrestrial $4 \times 2$ MIMO (SFN)}

The transceiver configuration of this scenario is similar to Scenario 2 except for the use of two antennas on each transmitter. The satellite uses LHCP and RHCP for the transmission, whereas CGC uses two linear polarizations. Since both SC and CGC use dual polarization, the UT also employs a dual polarized antenna, leading to a $4 \times 2$ MIMO configuration. It is again noted that only for a small percentage of the coverage will both the SC and the CGC be available. The literature for $4 \times 2$ codes is 
rich, but most of the $4 \times 2$ codes are not suitable for distributed transmitters with requirement of single link functionality. Some distributed $4 \times 2$ codes that are suitable for distributed hybrid satellite transmission are reported in $[4,8,9]$. The most straightforward transmission procedure is to send the same spatially multiplexed streams from satellite and terrestrial base station, and consider these signals as multipath components. Thus, both links have $2 \times 2$ spatial multiplexing, and the situation for the reception is equal to the case of single link $2 \times 2$ spatial multiplexing. This single layer coding scheme is referred here as distributed $4 \times 2$ MIMO SM. Another, a bit more complex, dual layer coding scheme for the $4 \times 2$ SFN configuration was introduced by adding Alamouti STBC across the two links while keeping the transmission of spatially multiplexed $2 \times 2$ streams on both links. This scheme is referred here as distributed $4 \times 2$ MIMO SM+block Alamouti.

\section{Scenario 4: hybrid satellite/terrestrial $4 \times 2 \mathrm{MIMO}(\mathrm{MFN})$}

Scenario 4 is quite similar to Scenario 3. The only difference being the MFN operation. This translates into a dual-polarization satellite transmitting in S-band and a dual-polarization CGC transmitting at UHF band. In fact, as the satellite and terrestrial links occupy different frequency bands, this scenario is equivalent to two independent $2 \times 2 \mathrm{MIMO}$ hybrid transmission schemes. The complementary code combining, where the turbo code bits of the two components are combined prior to turbo decoding, was utilized in combining the SC and CGC signals. The MIMO scheme selection for this scenario was performed according to scenario 1 .

\section{DESCRIPTION OF SIMULATION SYSTEM}

A MATLAB simulator with full physical layer functionality of DVB-SH was implemented. The SISO simulator was first programmed according to DVB-SH standard [11], and the MIMO-related functionalities were added as separate blocks as shown in Figures 2 and 3. The differences compared with SISO DVB-SH standard at the transmitter (Tx) side are the support for multiple parallel streams on common processing blocks, the MIMO encoding blocks and the modification of channel estimation pilots to support MIMO channel estimation for the OFDM option. For the TDM option, no modification to the existing DVB-SH standard is required. The required changes on the Rx side are respectively the MIMO detector block, the MIMO channel estimation block and the support for multiple parallel streams on the common processing blocks.

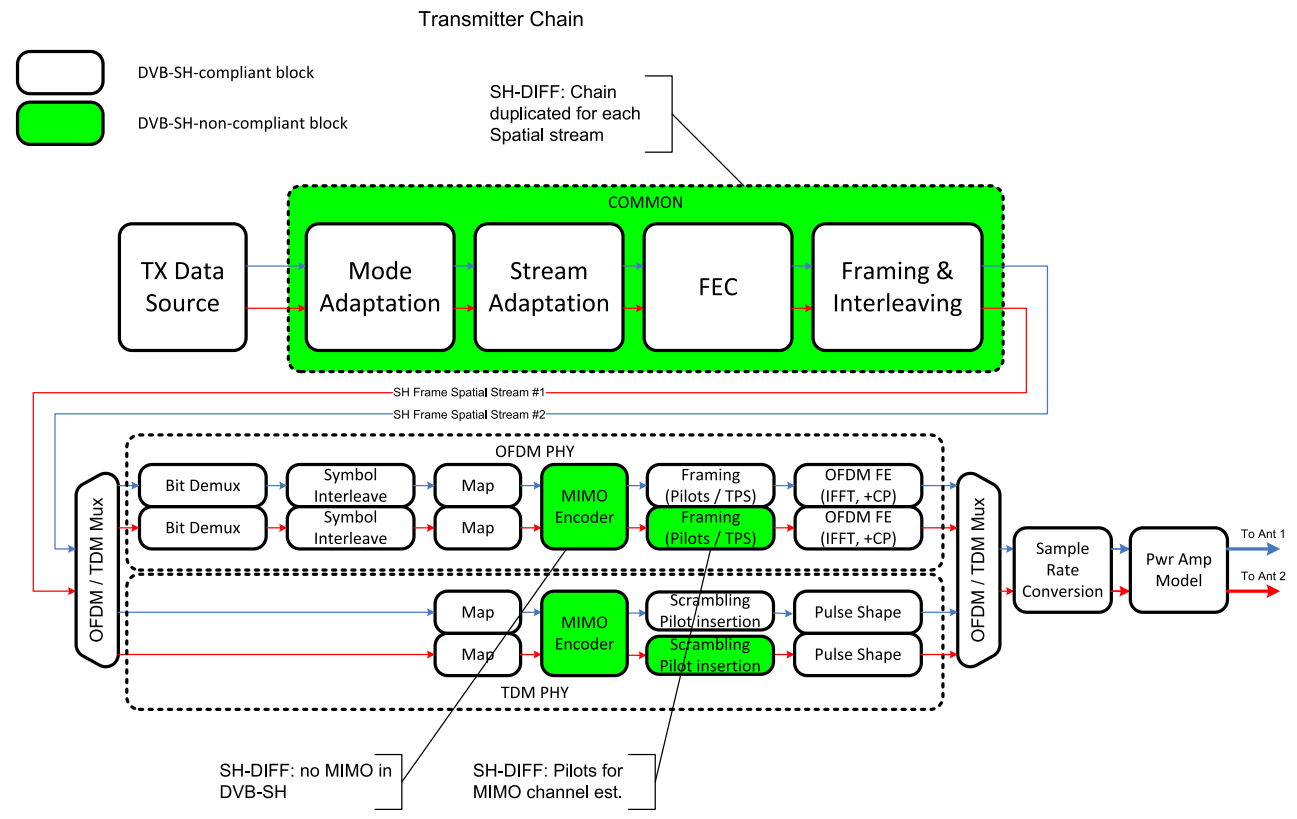

Figure 2. Simulator transmitter (TX)-chain block diagram. CP, cyclic prefix 


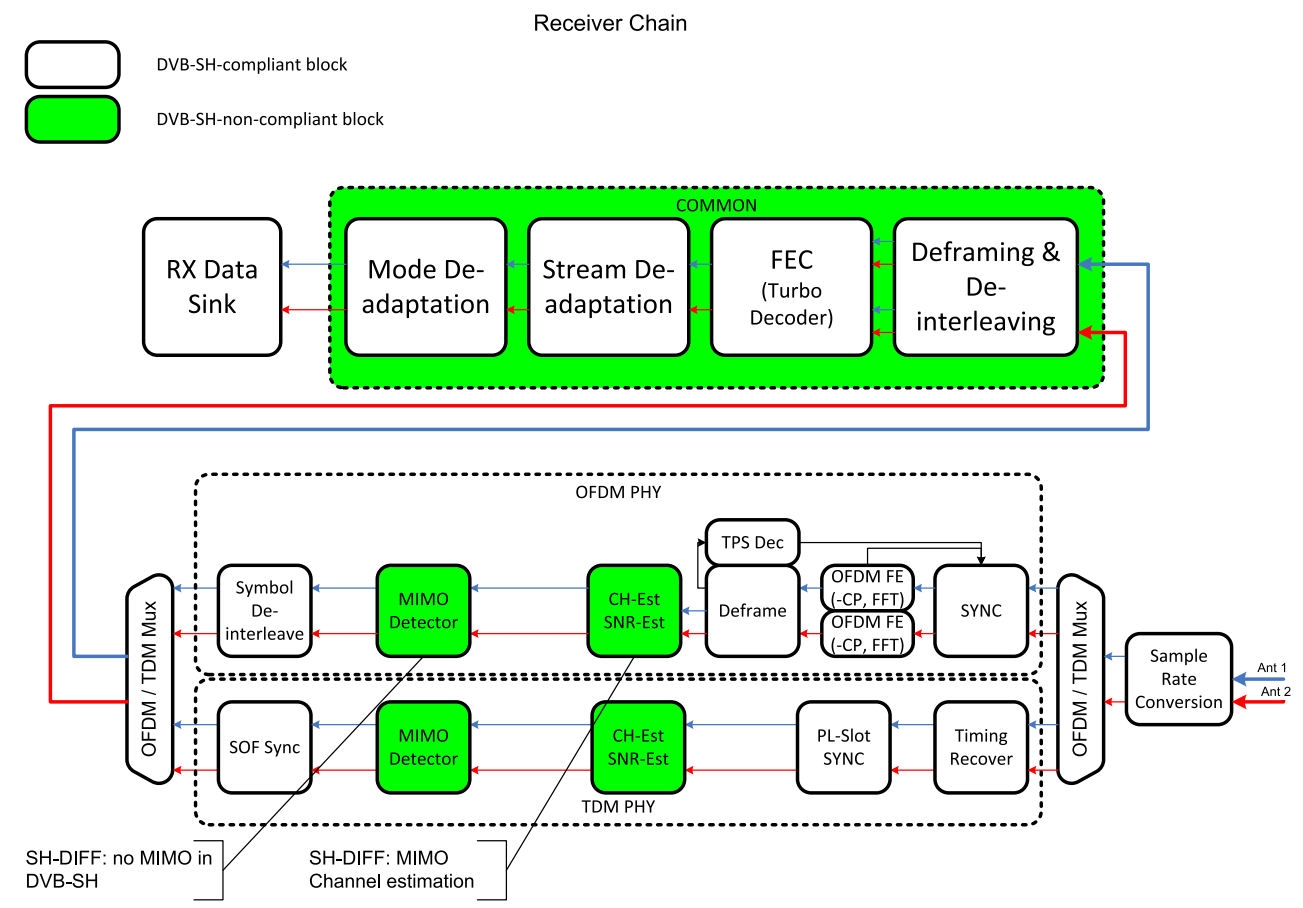

Figure 3. Simulator receiver (RX)-chain block diagram. SOF, start of frame; SNR, signal-to-noise ratio; $\mathrm{CH}$, channel; Ant, antenna.

The simulator blocks are similar for the satellite and terrestrial links and the only difference in the simulation is the radio channel. The most critical DVB-SH specific simulator blocks that affect the performance are those for the FEC and the time interleaving. The turbo code as standardized by the 3GPP2 organization is used as FEC coding, and it was implemented using the max-log-maximum-a-posteriori algorithm.

The MIMO extension for the Tx chain was implemented based on the standardized SISO simulator by adding the MIMO encoder block and modifying the scattered pilots of antenna 2 channel in order to enable MIMO channel estimation at the receiver. The functionality of common processing blocks is similar as for SISO but the multiple parallel stream support was implemented by introducing the parallel FEC and time interleaving blocks. The SH-A pilot allocation scheme of the SISO standard was retained for antenna 1 , and in the case of multiple Tx-antennas, the pilots of the other antennas were sent on the overlapping slots. The orthogonality of the pilot subcarriers is obtained by assigning cyclical phase shifts for the overlapping pilot sequences. By using this method, the pilot overhead is kept in a minimum as well as an adequate number of pilots in frequency domain is guaranteed also for the frequency selective terrestrial channel estimation. The implementation of the SH-A channel estimation is based on a method described in [12]. The SH-B channel estimation was implemented by employing existing semi-orthogonal scrambling sequences for the additional antennas and using the maximum likelihood feed-forward channel estimator algorithm described in the ETSI documentation [10]. The soft bit log-likelihood ratio (LLR) decoding requires the MIMO scheme specific implementation at the receiver. The SISO reception was implemented as a single symbol maximum-likelihood decoder. The Alamouti schemes employ the same SISO decoder after the Alamouti code demapping by channel orthogonalization. The more demanding MIMO schemes, that is, SM, golden code and the $4 \times 2$ schemes employ a list sphere decoder (LSD) receiver for the LLR extraction [17].

\section{CHANNEL MODELS}

An important aspect in any MIMO system simulation or emulation is that the underlying radio channel model is as realistic as possible. The system performance is strictly related to radio channel properties, especially the signal fading characteristics and the degree of correlation between the antennas at the 
transmitter and the receiver. Furthermore, the model shall include polarization effects because analytical and experimental work carried out on LMS channels at L- and S- frequency bands focuses in most cases on exploiting polarization diversity at both the transmitter and receiver when forming a $2 \times 2$ MIMO matrix. The dual-polarized antenna is extremely suitable for satellite use because the space limitation of a single satellite cannot provide the adequate antenna spacing required for the low degree of antenna correlation that is needed by MIMO theory. Recently, a statistical model for dual-polarized $2 \times 2$ MIMO LMS channels has been proposed, which is based on SISO LMS and MIMO wireless radio channel experimental results and their extrapolation to LMS channels [13]. The model is partly based on the SISO LMS channel model [14], which has been applied and validated in the standardization activity of DVB-SH. It models all the relevant channel characteristics including the user environment, the elevation angle of the satellite, the shadowing of the LOS signal component, the impact of temporal and polarization correlations, the antenna's cross-polar discrimination (XPD), and the environment's XPR. In addition, the model introduces a step-by-step approach for efficient time-series generation of dual-polarized $2 \times 2$ MIMO LMS channel coefficients. Therefore, representing state-of-the-art channel modeling for DVB-SH satellite systems, it is especially suitable for the current study.

In the following, we summarize the $2 \times 2$ MIMO LMS radio channel parameters applied in this study. The parameters were chosen to be the same as those employed by the Satellite Task Force of DVB-NGH [6]. The intermediate tree shadowing propagation environment was applied in all the simulations presented in this paper. The applied dual-polarized channel characteristics are shown in Table I.

For the SISO and MIMO terrestrial channel model, we have applied the 6-tap typical urban 6 (TU6) channel model of COST207 and the WINNER II channel model, respectively [16]. The WINNER II channel model follows a geometry-based stochastic channel modeling approach, which allows creating of an arbitrary double directional radio channel model with desired spatiotemporal and polarization properties. The channel model is antenna independent, which enables the insertion of different antenna configurations with different antenna patterns into the model. The channel parameters are determined stochastically, based on statistical distributions extracted from channel measurements. The applied TU6 radio channel parameters are summarized in Table II.

\section{SIMULATION RESULTS}

The simulation results for each scenario are presented in this section. The results are represented here for the SH-A (OFDM) scheme with the LMS-intermediate tree shadowing channel model. The physical layer system settings of Table III were applied in the simulations. The link-layer functionalities, such as iFEC were not taken into account. The performance metrics for the result comparison are the bit error rate and the erroneous second ratio (ESR)5(20) performance. The ESR5(20) is the ratio of windows for which ESR5 is fulfilled, over the total number of windows. The ESR5 criterion is fulfilled when in a time interval of $20 \mathrm{~s}$ there is at most one $1 \mathrm{~s}$ in error [10]. Evaluation of the ESR5(20) metrics is important for the satellite link video quality due to the long fading states of the LMS channel. The ESR5(20) fulfillment criteria for DVB-SH physical layer is $90 \%$. The MIMO schemes and modulation and coding schemes for the simulations were chosen to represent same spectral efficiency (data rate) for each case. The simulation schemes are shown in Table IV. All schemes provide double-spectral efficiency compared with SISO QPSK 1/3. Therefore SISO 16QAM 1/3, providing the same spectral efficiency with the MIMO schemes, was selected as a benchmark in order to observe the MIMO gain over SISO as a measure of signal-to-noise ratio (SNR). The SNR metric, $E_{\mathrm{S}} / N_{0}$, is defined as energy per symbol to noise ratio, and the total transmission power is kept equal for each case, thus the transmit power per transmit antenna in $2 \times 2$ MIMO case is half of the transmit power of the single-antenna transmit power in SISO case. The SNR is defined for the LOS-state of the LMS co-polarization channel. The total reception power over the four elements of $2 \times 2$ MIMO channel is slightly higher compared with SISO. The LMS channel XPR is quite high, and therefore, the total MIMO reception power by two orthogonally polarized antennas is only $0.67 \mathrm{~dB}$ higher than the SISO reception power in the simulation. A single-input multiple-output (SIMO) configuration with two antenna reception 
Table I. $2 \times 2$ multiple-input single-output land-mobile satellite radio channel channel parameters.

\begin{tabular}{|c|c|}
\hline Parameter & Value used \\
\hline Channel realization time & $\begin{array}{l}1 \mathrm{~h} \text { of simulation is the minimum to } \\
\text { guarantee stable ESR5(20) statistics. }\end{array}$ \\
\hline Carrier frequency & S-band $(2.2 \mathrm{GHz})$ \\
\hline Vehicle speed in kilometer per hour & 60 \\
\hline Polarization & RHCP/LHCP \\
\hline Propagation environment & ITS \\
\hline Elevation angle & 40 \\
\hline Small-scale correlation & \\
\hline $\begin{array}{l}\text { Correlation coefficient between polarizations } \\
\text { at the } \mathrm{Tx} / \mathrm{Rx}\end{array}$ & 0.5 \\
\hline Large-scale correlation & \\
\hline Correlation betweenLOS components & $0.85-0.93$ \\
\hline$X P D$ parameters & \\
\hline Antenna polarization discriminator in decibel: $\mathrm{XPD}_{\mathrm{ant}}$ & 15 \\
\hline Environment cross-polarization effects in decibel: $X P D_{e n v}$ & 5.5 \\
\hline 2-state first-order Markov model & \\
\hline Good $\rightarrow$ Good & 0.6 \\
\hline $\mathrm{Bad} \rightarrow \mathrm{Bad}$ & 0.8065 \\
\hline Good $\rightarrow$ Bad & 0.4 \\
\hline Bad $\rightarrow$ Good & 0.1935 \\
\hline Markov state update interval & $0.18 \mathrm{~s}$ (3 m movement) \\
\hline Loo distribution triplet & As in Tables III and IV of [15] \\
\hline Doppler spectrum & Low pass filter \\
\hline
\end{tabular}

ESR, erroneous second ratio; RHCP/LHCP, right-hand circular polarization/left-hand circular polarization; ITS, intermediate tree shadowing; Tx/Rx, transmitter/receiver; LOS, light of sight; XPD, cross-polar discrimination.

Table II. Terrestrial typical urban 6 model for suburban environment.

\begin{tabular}{lccc}
\hline Tap number & Dealy $(\mu \mathrm{s})$ & Average relative power $(\mathrm{dB})$ & Doppler spectrum \\
\hline 1 & 0.0 & 0.0 & Classic \\
2 & 0.1 & -1.5 & Classic \\
3 & 0.3 & -4.5 & Classic \\
4 & 0.5 & -7.5 & Classic \\
5 & 15.0 & -8.0 & Classic \\
6 & 17.2 & -17.7 & Classic \\
\hline
\end{tabular}

Table III. Simulation system settings.

\begin{tabular}{ll}
\hline Parameter & Value used \\
\hline Waveform & DVB-SH A (OFDM) \\
Channel bandwidth & $5 \mathrm{MHz}$ \\
FFT size & $2 \mathrm{~K}(2048)$ \\
Guard interval (GI) & $1 / 4$ \\
Receiver class & Class 2 \\
Interleaver type & Uniform Long \\
Interleaver length & $\sim 10 \mathrm{~s}$ \\
Synchronization & Ideal \\
\hline
\end{tabular}

on same polarization would provide a higher total reception power than the $2 \times 2 \mathrm{MIMO}$ with orthogonal polarizations, but the usage of SIMO instead of SISO as a reference is not reasonable because of the co-location requirement of the reception antennas. Anyhow, SIMO may be considered as an additional level of diversity on top of the polarization domain whenever antenna diversity can be accommodated in the UT (e.g., in vehicular terminals). 
Table IV. Transmission schemes of scenario 1.

MIMO and modulation and coding schemes for scenario 1
Benchmark: SISO 16QAM $1 / 3$
2xSISO QPSK 1/3
MIMO Alamouti 16QAM 1/3
MIMO SM QPSK 1/3
MIMO Golden QPSK 1/3

The simulation results of scenario 1 are presented in Figure 4. These simulations were performed with ideal channel estimation and synchronization at the receiver. The advantage of MIMO compared with SISO is clearly seen from the results, and the gains are summarized in Table V. According to the ESR5(20) fulfillment criteria, the SM is clearly the best performing MIMO scheme. The SM gives almost $3 \mathrm{~dB}$ gain compared with SISO.

The Alamouti scheme was simulated both as STBC and space-frequency block code (SFBC) implementation. If STBC is employed in OFDM system, the Alamouti code block extends over instantaneous OFDM symbols in time domain. In case of SFBC, the Alamouti coding is performed over instantaneous subcarriers of each OFDM symbol in frequency domain, and the code block does not extend over time domain symbols. It was noted that due to long OFDM symbol duration of DVB-SH signal, the channel response does not remain constant over the duration of two symbol times of the Alamouti STBC code block if the receiver speed is high. The reception of Alamouti scheme is based on assumption of stationary channel response during code block. Each symbol is decoded separately after channel orthogonalization. One of the major advantages of the Alamouti scheme is its low complexity single-symbol decodability. Orthogonalization causes interference between symbols if channel response changes during the two instantaneous symbols of Alamouti code block. In order to avoid this problem, the joint decoding of two symbols for each symbol time can be applied at the receiver. However, this requires far more complex receiver, which is not a reasonable requirement for Alamouti. On the other hand, the LMS channel is expected to have a narrow delay dispersion, and therefore, its variation over frequency domain remains low. The channel response over neighboring OFDM frequency bins may be assumed to be close to constant. Therefore, the Alamouti coding was simulated for also $\mathrm{SFBC}$, and the performance at $60 \mathrm{~km} / \mathrm{h}$ receiver speed is clearly better than the STBC performance.

The performance of Golden code as a FR-FD code was expected to be close to equal or better compared with SM. As the goal for this experimentation is the development of hardware demonstrator, the receiver complexity was kept reasonable also in the simulations. Therefore, a LSD with list size of 32 was employed for the reception of the Golden code. The optimal list size for the $2 \times 2$
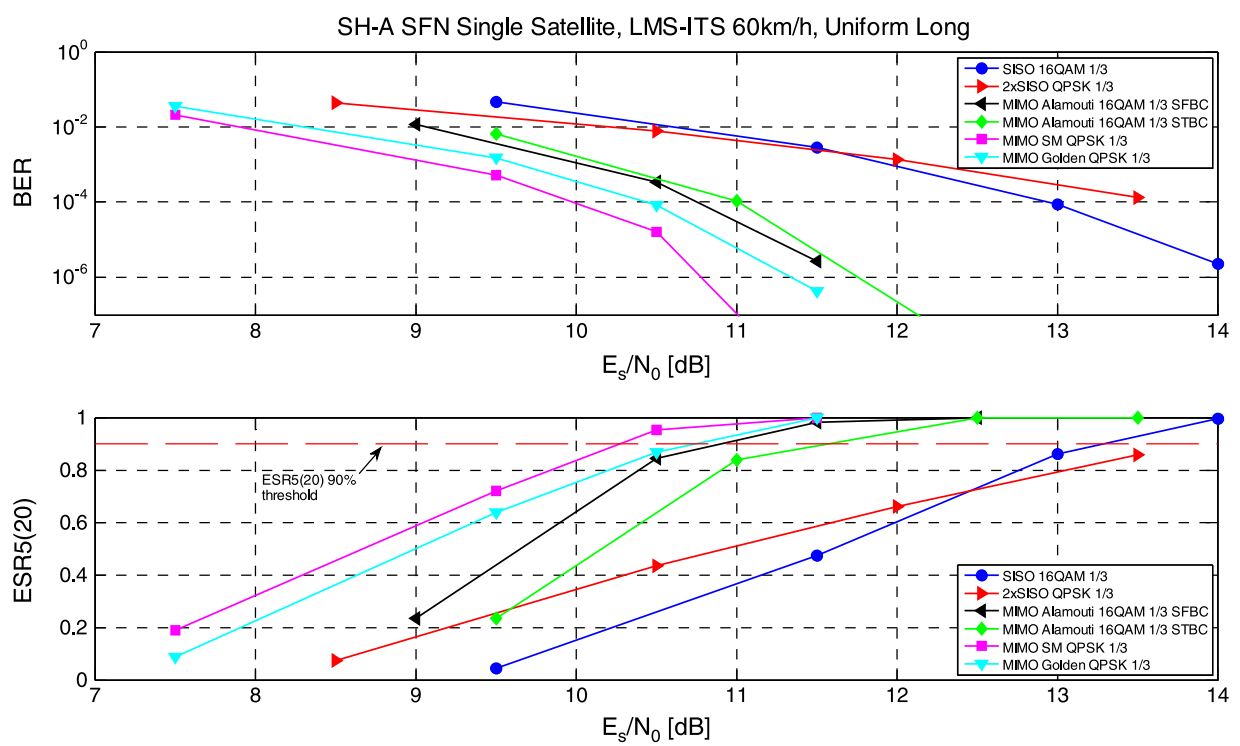

Figure 4. Scenario 1 simulation results. 
Table V. Scenario 1. Multiple-input single-output (MIMO) gain over single-input single-output (SISO).

\begin{tabular}{lcccc}
\hline \multicolumn{5}{c}{ SH-A MIMO scheme gain over SISO 16QAM 1/3 at 90\% ESR5(20) fulfillment level } \\
\hline SM & Golden & Alamouti SFBC & Alamouti STBC & 2xSISO \\
QPSK 1/3 & QPSK 1/3 & 16QAM 1/3 & 16QAM 1/3 & QPSK 1/3 \\
$2.9 \mathrm{~dB}$ & $2.6 \mathrm{~dB}$ & $2.4 \mathrm{~dB}$ & $1.7 \mathrm{~dB}$ & Approxim. $-0.5 \mathrm{~dB}$ \\
\hline
\end{tabular}

Golden code for QPSK is 256, and therefore, the poor performance of Golden code is partly explained by the non-ideal receiver. However, even for an optimal receiver, this code exhibits little improvement compared with what is reported here. The LSD was applied also for the reception of SM. But in this case, the applied list size was equal to the optimum list size for $2 \times 2$ QPSK, that is, 16. However, the performance gap between the SM and Golden code cannot be fully explained by the non-ideal reception. According to test simulations, increasing list size beyond 32 does not provide much performance gain. The reasons for the performance cap are the applied strong channel coding and interleaving, which decreases the effect of the diversity gain offered by Golden code. Also the LMS-channel model is known to be challenging for the FR-FD codes because of its high XPR and strong presence of LOS condition. The off-diagonal channel coefficients of the $2 \times 2$ dual-polarization channel matrix become weak because of the high XPR, and therefore, the power imbalance between the codeword components may deteriorate the performance of a FR-FD code.

The current DVB-SH standard does not contain any support for MIMO channel estimation in the case of the OFDM waveform. Therefore, a channel estimation scheme with minimum changes to existing DVB-SH pilot allocation scheme was implemented for the simulator. The effect of channel estimation and finite word-length MIMO detector was also studied by performing simulations with non-ideal channel estimation and fixed-point MIMO detector, and comparing the results to ideal simulation results. The simulation results of Figure 5 show that the performance loss due to non-ideal channel estimation and fixed-point detector is less than $0.5 \mathrm{~dB}$ for QPSK and less than $1 \mathrm{~dB}$ for the $16 \mathrm{QAM}$.

The scenario 2 simulations for $2 \times 1$ MISO hybrid were performed by using the LMS channel model for the satellite link and the TU6 suburban channel model for terrestrial link. The simulation results of Figure 6 show that adding the SC in addition to the CGC produces a gain of $1.25 \mathrm{~dB}$ at 90\% ESR5(20) fulfillment compared with terrestrial-only transmission. If the distributed $2 \times 1$ MIMO Alamouti STBC is used, an additional $0.25 \mathrm{~dB}$ gain was observed. It must be noted that the small gain compared with
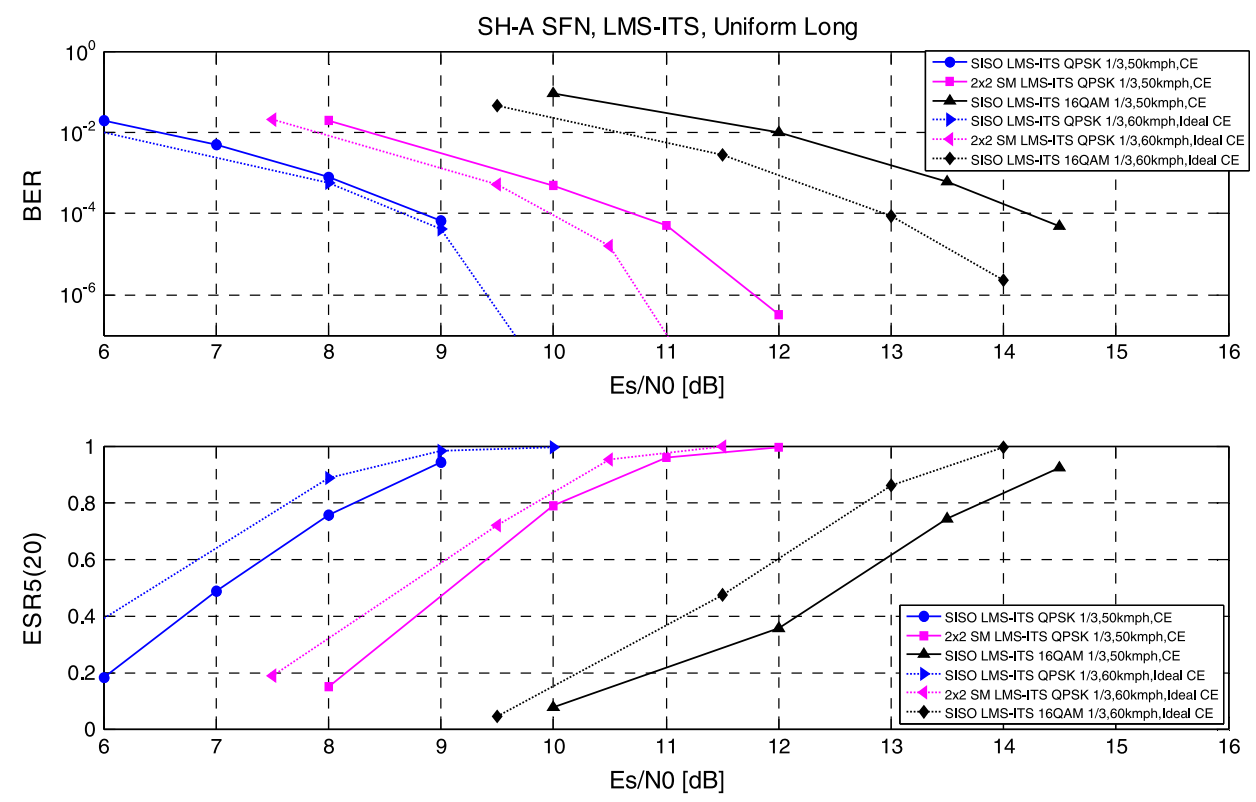

Figure 5. Comparison of ideal simulation results and simulations with non-ideal channel estimation and fixedpoint MIMO detector. CE, Channel Estimation; 

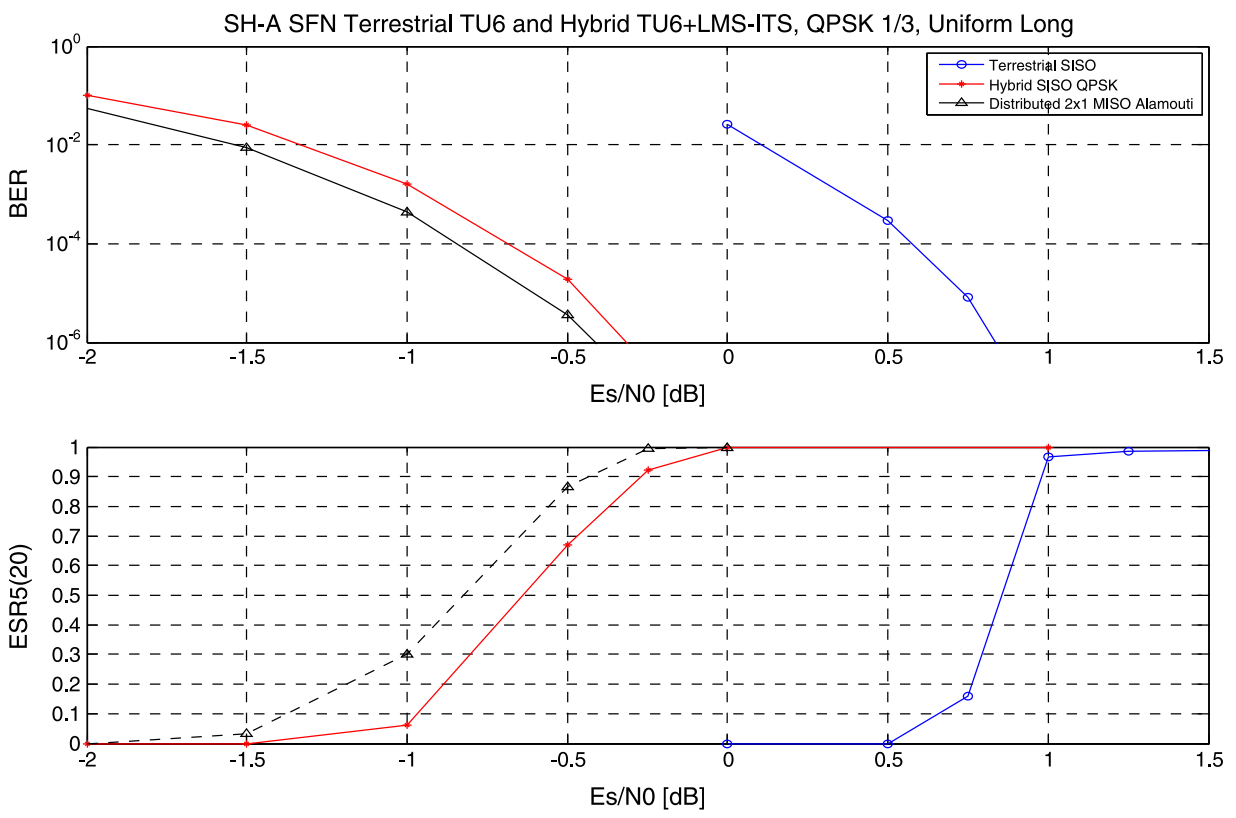

Figure 6. Scenario 2 simulation result for $2 \times 1$ multiple-input single-output (MISO).

terrestrial only is caused by the power imbalance between SC and CGC. In the case of Figure 6, the SC power is $3 \mathrm{~dB}$ lower than the CGC power.

The $2 \times 2$ MIMO hybrid simulations were performed for slightly different channel model configuration. The $1 \times 2$ terrestrial channel was applied by using WINNER II channel model for the simulation. The simulation result for $2 \times 2$ distributed Alamouti is shown in Figure 7. In this case, the addition of MIMO shows $2.3 \mathrm{~dB}$ gain over the normal $2 \times 1$ hybrid configuration.

The simulation results of scenario 3 are shown in Figure 8 . The gain of distributed $4 \times 2$ MIMO SM hybrid configuration compared with normal $2 \times 1$ hybrid is $4 \mathrm{~dB}$. It was observed that addition of Alamouti STBC over the links as a second layer coding does not bring notable performance gain compared with the simple single layer SM on both links. The complexity of the dual layer distributed $4 \times 2 \mathrm{SM}$ with block Alamouti is much higher as the maximum number of joint decoding symbol
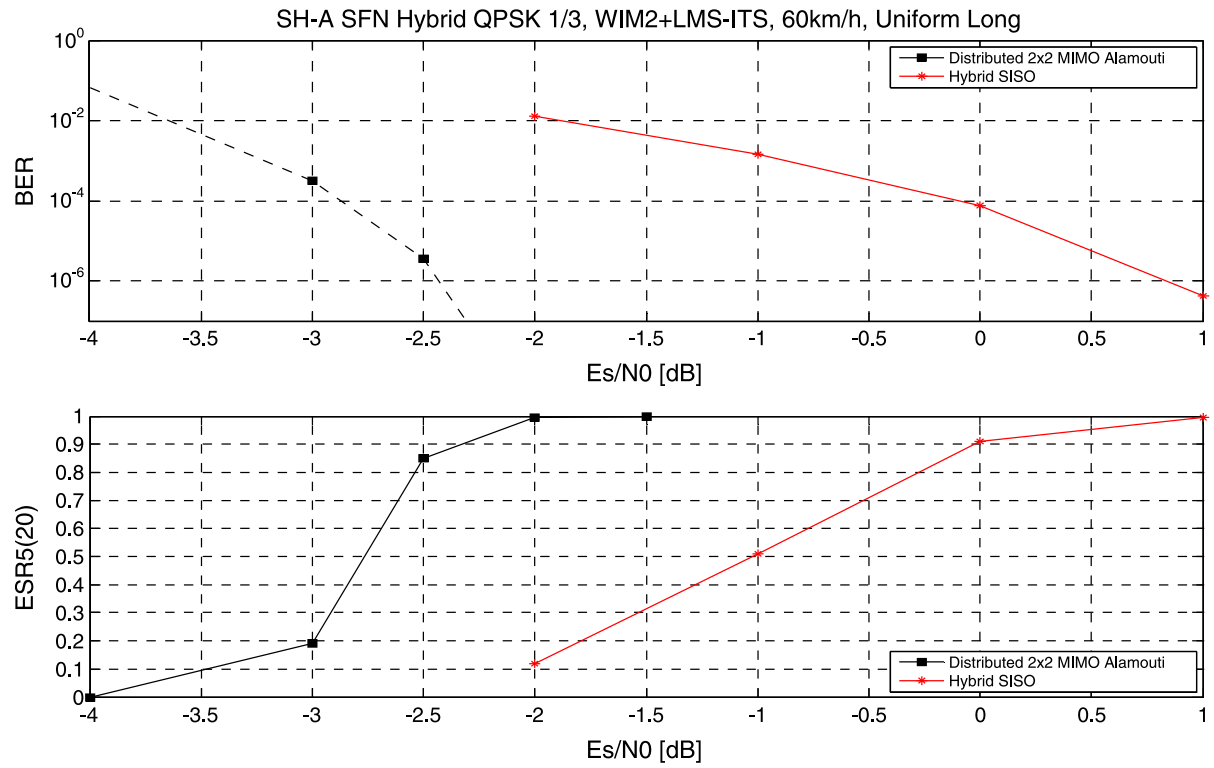

Figure 7. Scenario 2 simulation result for $2 \times 2$ distributed MIMO 

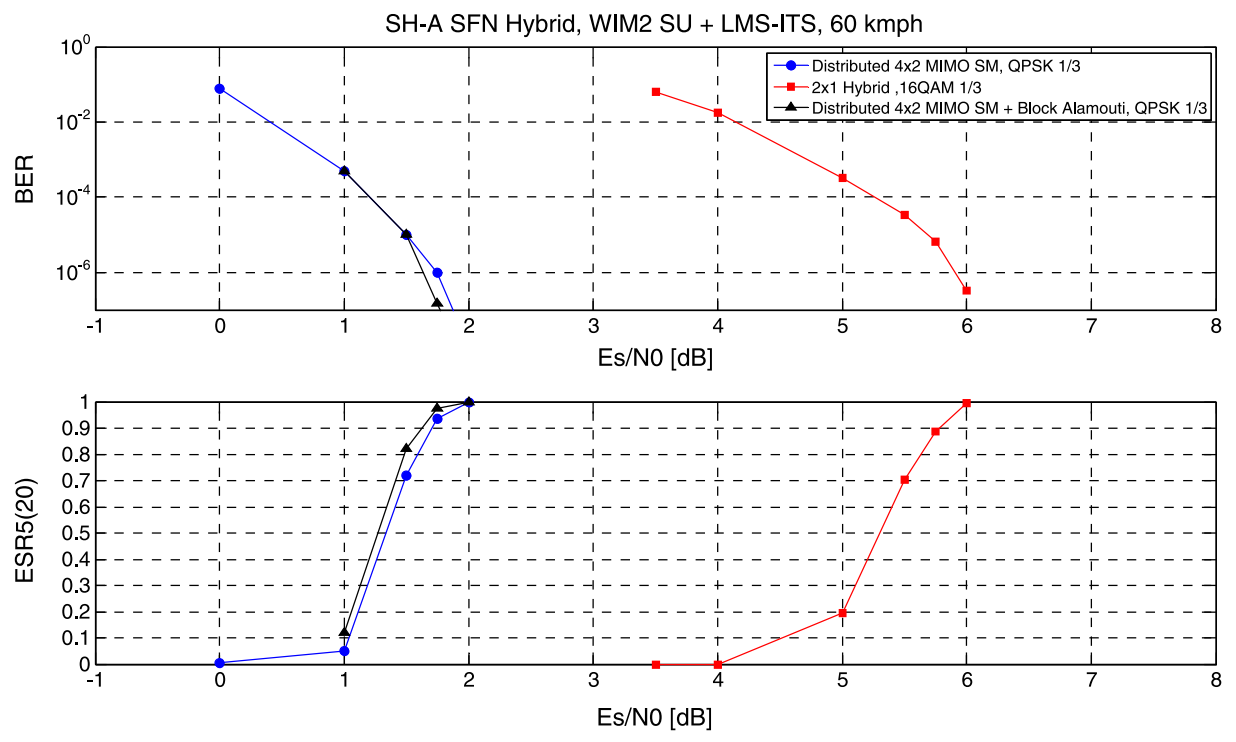

Figure 8. Scenario 3 simulation results.

candidates is 256 . The maximum number of decoding symbol candidates for the single layer distributed $4 \times 2$ MIMO SM is 16 . The list size of the LSD receiver used for the dual layer code was 32 . Thus, slightly higher gain for block Alamouti is possible if more complex receiver configurations are allowed.

The $4 \times 2$ hybrid configuration was also compared against $2 \times 2$ terrestrial MIMO link with SM in order to evaluate the advantage of hybrid configuration. The gain over $2 \times 2$ terrestrial SM was observed to be $4.5 \mathrm{~dB}$ if the satellite link reception power is equal to terrestrial link reception power and $1.5 \mathrm{~dB}$ if satellite link reception power is $4 \mathrm{~dB}$ weaker than terrestrial link reception power.

Scenario 4 was simulated using the same parameterization as Scenario 3. An important difference lies in the use of the UHF band for the terrestrial component. This translates into a different terrestrial channel parameterization with respect to earlier simulations. The available parameterization of the WINNER II model for the UHF band was employed for the terrestrial channel. Apart from the channel issue, the MFN configuration gives rise to the issue of how the receiver combines the received signals over the two distinct frequency bands. In the simulations presented herein, complementary code
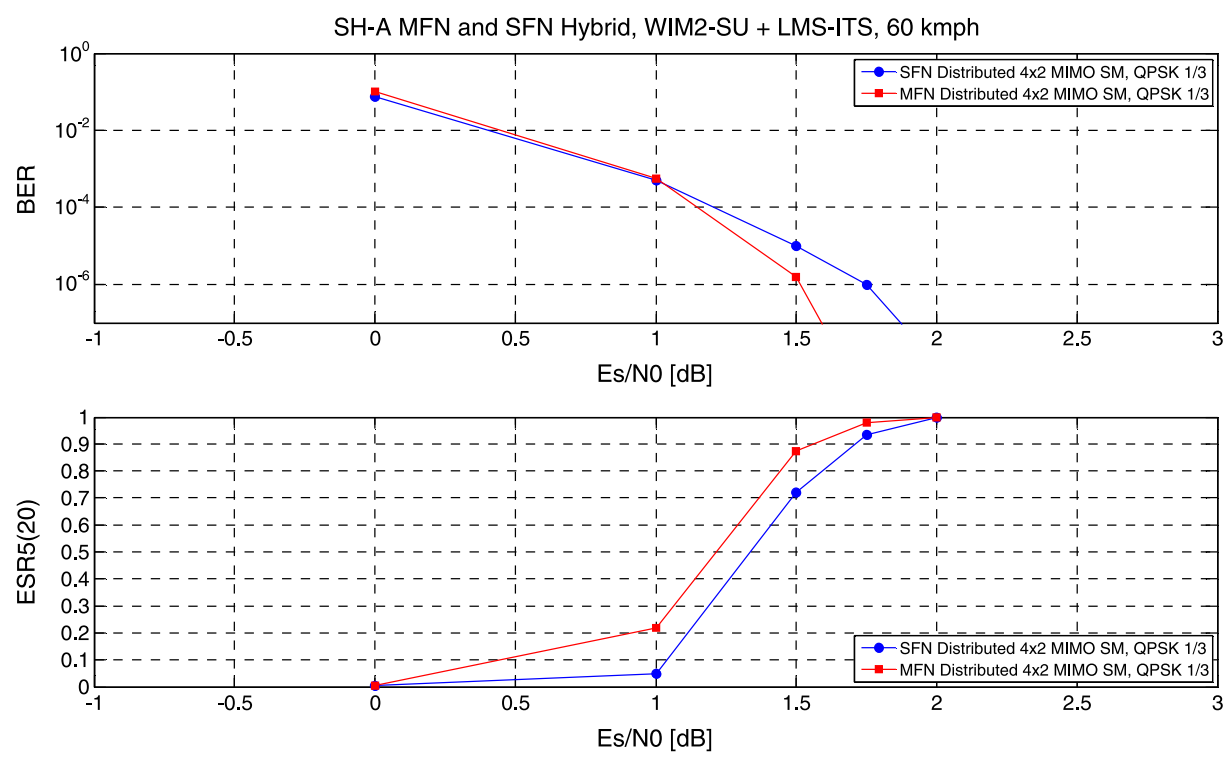

Figure 9. Scenario 4 simulation result. Comparison of multi-frequency network (MFN) and single-frequency network (SFN). 
combining has been employed as the combining technique. The distributed $4 \times 2$ MIMO SM hybrid MFN configuration is compared with the SFN configuration in Figure 9. The MFN configuration provides only $0.2 \mathrm{~dB}$ gain compared with SFN configuration. However, these results cannot be directly compared because of the different parametrization of the WINNER II channel model. The terrestrial link reception power of MFN configuration was $1.3 \mathrm{~dB}$ lower than in SFN case. However, it is obvious that doubling the bandwidth by employing two distinct bands for the hybrid transmission does not seem to give much advantage compared with SFN case.

\section{CONCLUSIONS}

Four potential satellite and hybrid MIMO scenarios for DVB-SH were described and analyzed in this paper. The evaluation of the potential MIMO scenarios, as well as the development of the DVB-SH MIMO simulator and analysis of the simulation results, forms a solid background for the development of the hardware demonstrator. Each of the MIMO scenarios were first analyzed based on the theory and available literature, and the most potential MIMO schemes were selected for each scenario. The performance of the selected MIMO schemes was simulated and analyzed in order to observe the advantages of MIMO in satellite communications and also to select the most promising schemes for hardware implementation and finally, for hardware emulations. The simulation results for the satellite-only transmission (scenario 1) showed reasonable gains for the MIMO schemes. The most promising techniques are SM and Alamouti SFBC, which were observed to provide $2.9 \mathrm{~dB}$ and $2.4 \mathrm{~dB}$ gain over the SISO reference scheme. SM was observed to provide good performance also in $4 \times 2$ hybrid scenario, where the multiplexing was applied for both $2 \times 2$ links. Addition of distributed Alamouti over the hybrid transmission links was found to be inefficient compared with the additional complexity that it requires in the decoding in case of the $4 \times 2$ hybrid scenario. On the other hand, if only one transmit antenna per link is available, and two receive antennas can be used in order to form $2 \times 2$ distributed MIMO hybrid, Alamouti was observed to provide $2.3 \mathrm{~dB}$ gain over normal $2 \times 1$ hybrid transmission. An approximate comparison between SFN and MFN was also presented for $4 \times 2$ hybrid MIMO. It was observed that doubling the bandwidth by using MFN configuration does not bring much advantage compared with SFN configuration.

\section{ACKNOWLEDGEMENTS}

The satellite MIMO study as well as the hardware demonstration development is carried out in co-operation with the European Space Agency under The MIMO Hardware demonstrator project, ESA AO/1-6099/09/NL/JK.

\section{REFERENCES}

1. Arapoglou P-D, Liolis K, Bertinelli M, Panagopoulos A, Cottis P, De Gaudenzi R, MIMO over satellite: a review. IEEE Communications Surveys Tutorials, $1^{\text {st }}$ Quarter, 2011.

2. Kim HW, Kang K, Ami DS. Distributed space-time coded transmission for mobile satellite communication using ancillary terrestrial component. Proceedings of IEEE International Conference on Communications, ICC 2007, June 2007, pp. 4426-4431.

3. Perez-Neira A, et al. MIMO channel modeling and transmission techniques for multi-satellite and hybrid satellite-terrestrial mobile networks. Elsevier's Physical Communication, 2011; 4(2), 127-139.

4. B. Shankar M.R, Arapoglou P-D, Ottersten B. Space-frequency coding for dual polarized hybrid mobile satellite systems. IEEE Transactions on Wireless Communications, 2012; 11:2806-2814.

5. Hollanti C, Raj Kumar K, Lahtonen J, Lu H-F, Vehkalahti R. Space-time block codes for the $2 \mathrm{Tx}+2 \mathrm{Rx}$ and $4 \mathrm{Tx}+2 \mathrm{Rx}$ antenna MIMO systems. DVB TM-H NGH Call for Technologies (CfT) proposal, February 2010.

6. ETSI EN 303105 Digital Video Broadcasting (DVB); Next generation broadcasting system to handheld, physical layer specification (DVB-NGH), 2012.

7. ITU-R WP4B, Annex 7 of Chairman Report, Reference network architectures, applications and performance, including QoS, for integrated systems operating within the mobile-satellite service (GEO) in the 1-3 GHz bands. 2010.

8. Hollanti C, Raj Kumar K, Lahtonen J, Lu. H-F, Vehkalahti R. Space-time block codes for the $2 \mathrm{Tx}+2 \mathrm{Rx}$ and $4 \mathrm{Tx}+2 \mathrm{Rx}$ antenna MIMO systems. DVB TM-H NGH Call for Technologies (CfT) proposal, February 2010.

9. Roivainen A, Kyröläinen J and Juntti M. New double layer space-time block code for distributed $4 \times 2$ MIMO systems. In Proc. IEEE Wireless Communications and Networking Conference (WCNC).1-4 April 2012, Paris, France, 232-235.

10. ETSI TS 102 584. Guidelines for implementation for satellite services to handheld devices (SH) below $3 \mathrm{GHz}$. June 2010.

11. ETSI EM 302 583. Digital video broadcast (DVB); framing structure, channel coding and modulation for satellite services to handheld devices (SH) below $3 \mathrm{GHz} .2007$. 
12. Y.Li, Simplified channel estimation for OFDM systems with multiple transmit antennas, IEEE Trans. On Wireless Comm. Vol.1 No.1, Jan.2002.

13. Liolis KP, Gomez-Vilardebo J, Casini E, Perez-Neira A. Statistical modeling of dual-polarized MIMO land mobile satellite channels. IEEE Transactions Communications, 2013; 58:3077-3083.

14. Fontán FP, Castro M-A V, Cabado C E, García J P, Kubista E. Statistical modeling of the LMS channel. IEEE Trans. Veh. Technol., 2001; 50:1549-1567.

15. Prieto-Cerdeira R, Perez-Fontan F, Burzigotti P, Bolea-Alamanac A, Sanchez-Lago I. Versatile two-state land mobile satellite channel model with first application to DVB-SH analysis. Int. J. Satell. Commun. Network., 2010; 28:5-6.

16. IST-WINNER II, D1.1.2 "WINNER II channel models", v.1.0, Sep 2007, https://www.istwinner.org/WINNER2-Deliverables/.

17. Myllylä M, Juntti M, Cavallaro J R. Implementation aspects of list sphere decoder algorithms for MIMO-OFDM systems. Signal Processing, 2010; 90(10).

\section{AUTHOR'S BIOGRAPHIES}

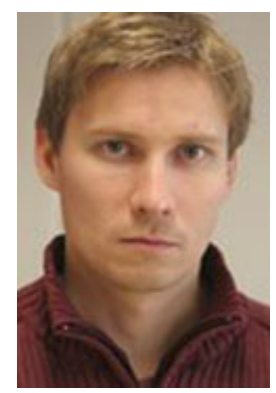

Jukka Kyröläinen received the MSc degree in electrical engineering from the University of Oulu, Oulu, Finland in 2007. During 2006-2011, he worked for the Centre for Wireless Communications in the University of Oulu as a Research Scientist. He joined Elektrobit (EB) in 2011 where he has been working as a Senior Design Engineer mainly in the areas of satellite communications and radio channel emulation applications. Since February 2013, he has continued his work in the field of radio channel research and emulation applications with Anite telecoms.

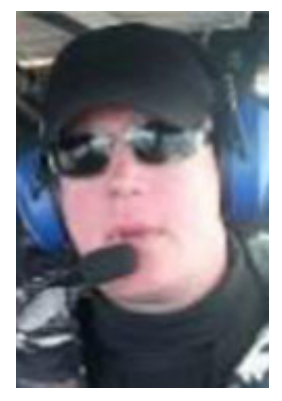

Ari Hulkkonen is a telecommunications professional who has worked for EB since 1994 as a radio frequency and system engineer and various management positions. With the strong background in wireless technologies and spread spectrum communications and years of working with industrial broadband communications applications, Hulkkonen was appointed to lead EB's research in 2001 with a goal to develop EB's know-how in MIMO and multicarrier technologies into a state-of-the-art level. Currently, he is continuing his work in coordinating waveform and technology development for special applications such as satellite and tactical communications with a special focus in cognitive radio technologies and software defined radio as the key implementation technology. As a part of his work with the special applications, he is leading the MIMO HW demonstrator project and is participating in both national and international research collaboration.

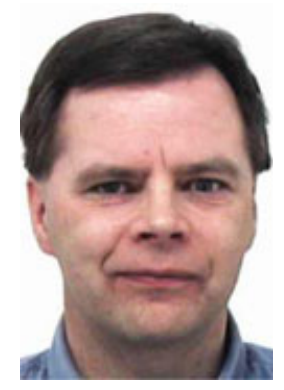

Juha Ylitalo received his MSc, Lic.Tech, and PhD Tech degrees in Electrical Engineering at the University of Oulu, Oulu, Finland in 1981, 1985, and 1987, respectively. During 19811994, he has held various research positions at the University of Oulu and the Academy of Finland studying digital synthetic aperture radar techniques. He was a visiting scientist in Rochester, MN, USA in 1985-1986 in Bochum, Germany 1991-1992, and in Trondheim, Norway 1994-1995. He joined Nokia Networks in 1995 where he was developing adaptive antenna techniques for CDMA (IS-95), GSM, and WCDMA base stations. He served Nokia as a specialist and a system program manager until 2004. Since 2004, he has been Research Manager and Senior Specialist at EB Corp. During 2004-2007, he acted also as a part-time Head of the 4G Lab (now called Wireless Networking Laboratory) at the Centre for Wireless Communications, University of Oulu.

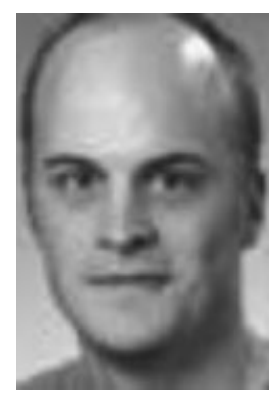

Aaron Byman received his BSEE from the University of Washington, USA in 1999 and MSEE from the University of Oulu, Finland in 2001. Byman is currently working in EB's research organization as a Senior Specialist with the main responsibility for air interface algorithm implementation in RACE-SDR environment. His areas of expertise include: MIMO, OFDMA, synchronization, MIMO-channel estimation and detection, and WCDMA technologies. 


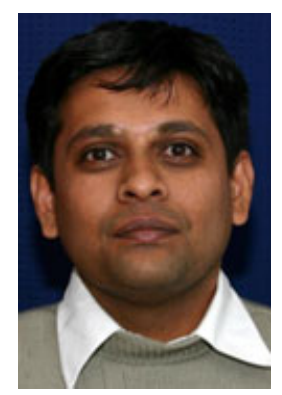

Bhavani Shankar M. R. received his Masters and $\mathrm{PhD}$ in Electrical Communication Engineering from the Indian Institute of Science, Bangalore in 2000 and 2007, respectively. He was received his post-PhD at the ACCESS Linnaeus Centre, Signal Processing Lab, Royal Institute of Technology (KTH), Sweden from 2007 to September 2009 and a Research Associate at Interdisciplinary Centre for Security, Reliability and Trust (SnT), University of Luxembourg from October 2009 to March 2013 where he now holds the position of a Research Scientist. He was with Beceem Communications (now acquired by Broadcom), Bangalore from 2006 to 2007 as a Staff Design Engineer working on Physical Layer algorithms for WiMAX compliant chipsets. He was a visiting student at the Communication Theory Group, ETH Zurich, headed by Professor Helmut Bölcskei during 2004. Prior to achieving his PhD degree, he worked on Audio Coding algorithms in Sasken Communications, Bangalore as a Design Engineer from 2000 to 2001. His research interests include Design and Optimization of MIMO Communication Systems, Satellite Communications, Resource Allocation, Game Theory, and Fast Algorithms for Structured Matrices.

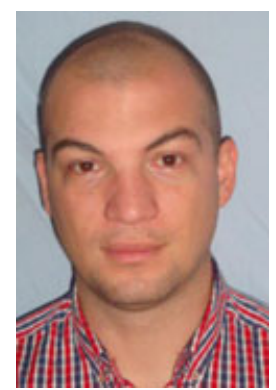

Pantelis-Daniel Arapoglou received the Diploma Degree in Electrical and Computer Engineering and his PhD Engineering Degree from the National Technical University of Athens (NTUA), Greece, in 2003 and 2007, respectively. From January 2004 until December 2005, he was a Research Assistant at the School of Pedagogical and Technological Education (ASPETE). From September 2005, he acted as a satellite coordination engineer for the Spectrum Management Division of the Hellenic Ministry of Transport and Communications. On August 2007, he began his mandatory military duty in the Electronic Warfare Corps of the Hellenic Army. From September 2008 to October 2010, he was involved in postdoctoral research on MIMO over satellite jointly supported by the NTUA and the European Space Agency Research and Technology Centre (ESA/ESTEC), The Netherlands. From October 2010 to September 2011, he was a Research Associate with the Interdisciplinary Centre for Security, Reliability, and Trust (SnT) of the University of Luxembourg. Since September 2011, he is a Communications System Engineer at ESA/ESTEC.

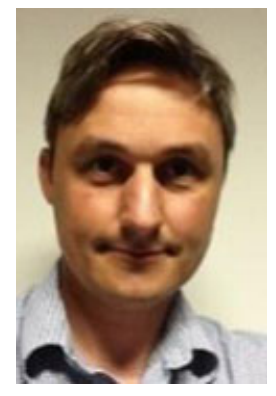

Joel Grotz graduated from the University of Karlsruhe and the Grenoble Institute of Technology and Electrical Engineering and from the Royal Institute of Technology (KTH), Stockholm with a ph.D. degree in Telecommunications. He has at this date over thirteen years of work experience in satelite communications. Within SES ASTRA and SES ENGINEERING in Luxembourg he worked on two-way satellite internet access systems, broadcast system designs as well as satellite payload design, specification and testing for satellite procurement assignments. Since November 2012, Joel Grotz is with the Technology Labs at NEWTEC in Belgium, working on modem and system development. In this role he is involved in DVB standardization activities and works on research projects focusing on new transmission techniques, standards and end-to-end systems for braodband and broadcast satellite systems. 